\title{
Presolar grains from meteorites: AGB star matter in the laboratory
}

\author{
Ernst Zinner and Sachiko Amari \\ McDonnell Center for the Space Sciences and Physics Department, \\ Washington University, St. Louis, MO 63130, USA
}

\begin{abstract}
Primitive meteorites contain dust grains that predate the Solar System, formed in stellar atmospheres and thus represent samples of ancient stardust. Among the presolar grain types identified so far, corundum $\left(\mathrm{Al}_{2} \mathrm{O}_{3}\right)$ and silicon carbide $(\mathrm{SiC})$ are inferred to originate from AGB stars. Corundum grains carry the signatures of core $\mathrm{H}$ burning in their $\mathrm{O}$ isotopes and of shell $\mathrm{H}$ burning during the AGB phase in the form of extinct ${ }^{26} \mathrm{Al}$. In presolar $\mathrm{SiC}$, most of which originated from carbon stars, the $\mathrm{C}$ and $\mathrm{N}$ isotopes and ${ }^{26} \mathrm{Al}$ reflect core and shell $\mathrm{H}$ burning and shell He burning. In addition, many elements that carry the isotopic signature of neutron capture have also been measured. Most individual grains show excesses in ${ }^{29} \mathrm{Si}$ and ${ }^{30} \mathrm{Si}$, but the contribution from neutron capture is only a minor effect and the major effect is due to galactic heterogeneity. Noble gases and the elements $\mathrm{Ba}, \mathrm{Nd}, \mathrm{Sm}$, and $\mathrm{Dy}$ are measured in "bulk samples", collections of many grains. Their measured isotopic patterns are well reproduced by models of the s-process in AGB stars. Recently, the isotopic analysis of $\mathrm{Sr}, \mathrm{Zr}$ and $\mathrm{Mo}$ in single $\mathrm{SiC}$ grains has been made possible by resonance ionization mass spectrometry. These measurements also point to low-mass AGB stars as the most likely sources. Specifically, large ${ }^{96} \mathrm{Zr}$ depletions in some grains indicate that the ${ }^{22} \mathrm{Ne}(\alpha, \mathrm{n})$ source was not active in the grains' parent stars.
\end{abstract}

\section{Introduction}

Until recently it was believed that the only samples of extrasolar matter that could be studied in terrestrial laboratories were galactic cosmic rays, and even those are quite elusive. Information about distant stars was mostly obtained in the form of electromagnetic radiation. This situation changed fundamentally when it was discovered that primitive meteorites contain presolar dust grains (Lewis et al. 1987; Bernatowicz et al. 1987), specks of stardust that formed in expanding stellar atmospheres and supernova ejecta and survived the formation of the Solar System. These grains can be isolated from the meteorites and studied in detail in the laboratory, providing new and important information on nucleosynthesis and stellar evolution, mixing in AGB stars and supernova explosions, condensation in stellar envelopes, and the chemical evolution of the Galaxy (Anders \& Zinner 1993; Ott 1993; Bernatowicz \& Zinner 1997; Zinner 1998). 


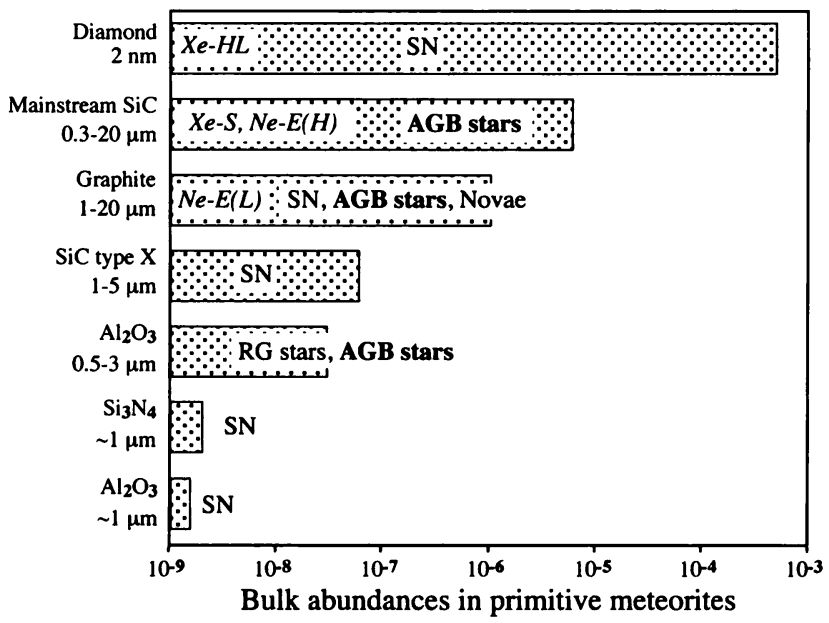

Figure 1. Abundances and sizes of presolar dust grains extracted from primitive meteorites. Inferred stellar sources and names of the anomalous noble gases carried by the grains are also indicated.

Fig. 1 lists the types of presolar grains discovered to date, together with their sizes, abundances, and likely stellar sources. Also given are the anomalous (or "exotic") noble gas components that are carried by some grain types. The presence of these noble gas components led to the discovery of presolar grains (see e.g., Anders \& Zinner 1993, Tang et al. 1988). We note that all grain types are high temperature condensates. This and, even more so, the fact that the isotopic compositions of essentially all the elements that could be measured in the grains are completely different from those found in the Solar System leave little doubt that these grains represent true stellar material and preserved the isotopic compositions of their celestial sources. With the exception of diamond, presolar grains are large enough that isotopic ratios can be measured in individual grains, mostly by ion microprobe mass spectrometry (Hoppe \& Ott 1997; Nittler 1997; Zinner 1998), recently also by micro-RIMS (resonance ionization mass spectrometry) (Nicolussi et al. 1997). Single grain measurements have revealed huge variations, which, for the elements $\mathrm{C}$ and $\mathrm{O}$, range over almost four orders of magnitude. Presolar grains thus yield information on the isotopic compositions of individual stellar sources.

Although stellar sources have to be inferred from the isotopic compositions of the grains, this can usually be done fairly reliably, especially in cases where many different elements can be analyzed. Two types of grains are inferred to have originated from AGB stars: the majority of corundum $\left(\mathrm{Al}_{2} \mathrm{O}_{3}\right)$ and silicon carbide (SiC) grains. A small fraction $(\sim 1 \%)$ of each of these presolar grain types apparently comes from supernovae (see Fig. 1), however we will not discuss those. An AGB origin of graphite grains rests mainly on noble gas analysis (Amari et al. 1995) and diagnosis is less certain for individual grains. 


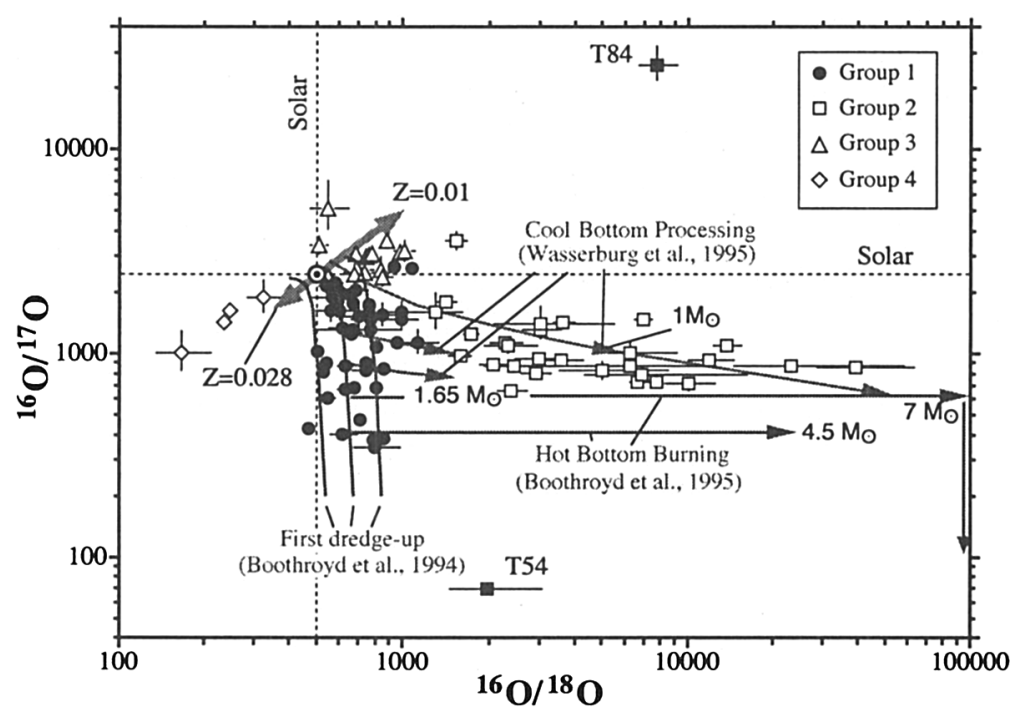

Figure 2. Oxygen isotopic ratios measured in single corundum grains. Four groups are distinguished by their isotopic ratios (Nittler et al. 1997), grains T54 and T84 are ungrouped. Also indicated are processes (CNO burning and first dredge-up, hot bottom burning, and cool bottom processing) that change the $\mathrm{O}$ isotopic ratios on the surface of $\mathrm{RG}$ and AGB stars. Arrow from $\mathrm{Z}=0.01$ to $\mathrm{Z}=0.028$ indicates the range of $\mathrm{O}$ ratios expected for this metallicity range. Broken lines in this and subsequent figures depict solar isotopic ratios.

\section{Corundum grains}

A little more than 100 presolar corundum grains have been identified to date and analyzed for their $\mathrm{O}$, a fraction also for their $\mathrm{Mg}-\mathrm{Al}$ and $\mathrm{Ti}$ isotopic compositions (Hutcheon et al. 1994; Nittler et al. 1997; Choi et al. 1998b, 1998a). Because presolar corundum in the size range indicated in Fig. 1 constitutes only $\sim 1 \%$ of meteoritic corundum grains (most are of Solar System origin and are isotopically normal), isotopic imaging in the ion microprobe that allows the automatic analysis of many grains plays a crucial role in the identification of anomalous grains (Nittler et al. 1997).

Fig. 2 shows the $\mathrm{O}$ isotopic ratios measured in most presolar oxide grains. Nittler et al. (1997) divided the grains into four groups according to their O isotopic ratios. Grains of group 1 and 3 show the signature of $\mathrm{H}$ burning via the $\mathrm{CNO}$ cycle in low- and intermediate-mass stars and subsequent mixing of core material into the envelope by the first (in more massive stars also the second) dredge-up (Dearborn 1992; Boothroyd et al. 1994; El Eid 1994; Lattanzio $\&$ Boothroyd 1997). These processes result in ${ }^{17} \mathrm{O}$ enrichments and moderate ${ }^{18} \mathrm{O}$ depletions. Variations in the ${ }^{16} \mathrm{O} /{ }^{17} \mathrm{O}$ ratio mostly reflect differences in stellar mass whereas variations in the ${ }^{16} \mathrm{O} /{ }^{18} \mathrm{O}$ ratio can only be explained by differences in the original isotopic ratios of the stars, corresponding to different 
metallicities according to a galactic evolution model (Timmes et al. 1995). The three lines labeled "First dredge-up" in Fig. 2 depict the predicted ratios for stars of different masses and metallicities (Boothroyd et al. 1994, Boothroyd \& Sackmann 1998). Giant stars with a range of masses and metallicities must have contributed oxide grains to the Solar System and the O isotopic ratios of group 1 and especially group 3 grains provide information on the galactic evolution of the $\mathrm{O}$ isotopes (Nittler 1997).

In addition to agreeing with theoretical expectations, the $\mathrm{O}$ isotopic compositions of group 1 and 3 grains are similar to those observed in red giant and AGB stars (Harris \& Lambert 1984; Harris et al. 1987; Smith \& Lambert 1990). AGB stars are the more likely sources since the large mass loss such stars undergo is expected to favor the formation of grains. Additional information that lets us distinguish between RG and AGB stars is provided by the presence of large ${ }^{26} \mathrm{Mg}$ excesses from the decay of ${ }^{26} \mathrm{Al}$ in many grains. More than half of group 1 and 3 grains have such excesses; inferred ${ }^{26} \mathrm{Al} /{ }^{27} \mathrm{Al}$ ratios range up to 0.01 (Nittler et al. 1997). Since ${ }^{26} \mathrm{Al}$ is produced at the higher temperatures characteristic of shell burning of $\mathrm{H}$ (Forestini et al. 1991), an AGB origin is indicated at least for these grains.

Group 2 grains have large ${ }^{18} \mathrm{O}$ depletions that cannot be explained as the result of first and second dredge-up because such an explanation would require unreasonably low metallicities. Hot bottom burning in AGB stars with $M>4 \mathrm{M}_{\odot}$ would effectively destroy ${ }^{18} \mathrm{O}$, but such stars are predicted to have lower ${ }^{16} \mathrm{O} /{ }^{17} \mathrm{O}$ ratios than those observed in group 2 grains (Boothroyd et al. 1995). However, a model of slow circulation of envelope material through hot regions near the H-burning shell ("cool bottom processing") successfully reproduces the group 2 oxygen data (Wasserburg et al. 1995; Denissenkov \& Weiss 1996).

Although an AGB origin either from stars with very high metallicities or from stars with ${ }^{18} \mathrm{O}$ dredge-up during early thermal pulses (Boothroyd \& Sackmann 1988) has been considered for group 4 grains, a $\mathrm{SN}$ origin has also been proposed (Choi et al. 1998a). Grain T84 with its large ${ }^{16} \mathrm{O}$ excess is definitely a SN candidate (Nittler et al. 1998), and T54 possibly comes from a more massive AGB star with hot bottom burning (Nittler et al. 1997).

\section{Light elements in presolar $\mathrm{SiC}$}

In contrast to corundum, all meteoritic $\mathrm{SiC}$ appears to be of presolar origin and most of it is believed to originate from carbon stars because: 1 . The distribution of ${ }^{12} \mathrm{C} /{ }^{13} \mathrm{C}$ ratios of single $\mathrm{SiC}$ grains is similar to that observed in carbon stars (see Fig. 3 in Bernatowicz \& Walker 1997); 2. AGB stars are the main contributors of carbonaceous dust to the interstellar medium (Whittet 1992); 3 . Circumstellar dust shells of carbon stars show the $11.2 \mu \mathrm{m}$ emission line of $\mathrm{SiC}$ (Little-Marenin 1986); and 4. SiC grains carry s-process isotopic signatures in the heavy elements (Hoppe \& Ott 1997) and AGB stars are believed to be the major source of the s-process elements (Gallino et al. 1998).

Fig. 3 shows the $\mathrm{C}$ and $\mathrm{N}$ isotopic ratios measured in single $\mathrm{SiC}$ grains. Based on the $\mathrm{C}, \mathrm{N}$, and $\mathrm{Si}$ isotopes, several groups can be distinguished (Hoppe $\&$ Ott 1997, Zinner 1998). The mainstream grains account for $\sim 95 \%$ of all grains. They and most $\mathrm{Y}$ and $\mathrm{Z}$ grains probably come from AGB stars (Zinner 


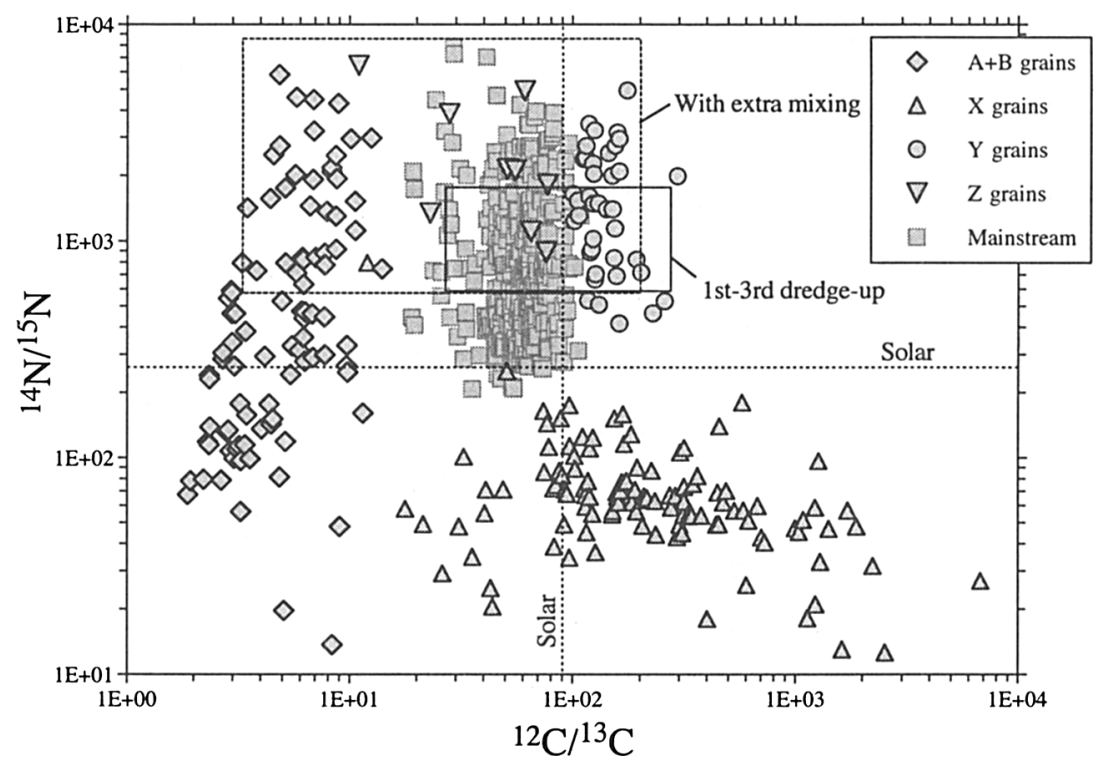

Figure 3. Carbon versus nitrogen isotopic ratios measured in single SiC grains from the Murchison carbonaceous meteorite. Five groups can be distinguished on the basis of the $\mathrm{C}, \mathrm{N}$, and $\mathrm{Si}$ isotopic ratios. Also indicated are the theoretically expected ranges resulting from $\mathrm{H}$ and $\mathrm{He}$ burning and subsequent dredge-up and from extra mixing (cool bottom processing) in lowto intermediate-mass carbon stars.

$1998)$ and only $\mathrm{SiC}$ grains of type $\mathrm{X}(\sim 1 \%)$, characterized by ${ }^{15} \mathrm{~N}$ and ${ }^{28} \mathrm{Si}$ excesses, are inferred to have a SN origin (Nittler et al. 1995). Note that the distribution of the grains in Fig. 3 does not correspond to their natural abundances but that, because of special searches by ion imaging, rare grain types are overrepresented. The $\mathrm{C}$ and $\mathrm{N}$ isotopic ratios of most mainstream grains are generally in agreement with theoretical expectations for carbon stars (CNO cycle during core $\mathrm{H}$ burning followed by first and second dredge-up and shell $\mathrm{He}$ burning followed by the third dredge-up) (Iben \& Renzini 1983; Bazan 1991; El Eid 1994; Frost \& Lattanzio 1996). This is especially the case if extra mixing (cool bottom processing) is taken into account (Charbonnel 1995, Wasserburg et al. 1995). However, low ${ }^{14} \mathrm{~N} /{ }^{15} \mathrm{~N}$ ratios, especially in type $\mathrm{A}+\mathrm{B}$ grains, which have low ${ }^{12} \mathrm{C} /{ }^{13} \mathrm{C}$ ratios, still do not have a satisfactory explanation (Hoppe et al. 1996; Amari et al. 1997; Huss et al. 1997). We will not discuss these any further.

Most $\mathrm{SiC}$ grains of inferred AGB origin have large ${ }^{26} \mathrm{Mg}$ excesses from the decay of ${ }^{26} \mathrm{Al}$ (Hoppe et al. 1994; Huss et al. 1997) and bulk samples have large ${ }^{22} \mathrm{Ne}$ excesses (Lewis et al. 1990, 1994). ${ }^{26} \mathrm{Al}$ is produced by'shell $\mathrm{H}$ burning and is mixed into the envelope by the third dredge-up (Forestini et al. 1991) while ${ }^{22} \mathrm{Ne}$ is produced from ${ }^{14} \mathrm{~N}$ by shell He burning. Ne isotopic ratios in $\mathrm{SiC}$ are very similar to those predicted for He-shell material (Gallino et al. 1990).

In contrast to $\mathrm{C}$ and $\mathrm{N}$, the $\mathrm{Si}$ isotopes are not much affected by $\mathrm{H}$ burning but mostly by neutron capture in the He-burning shell (Gallino et al. 1990, 


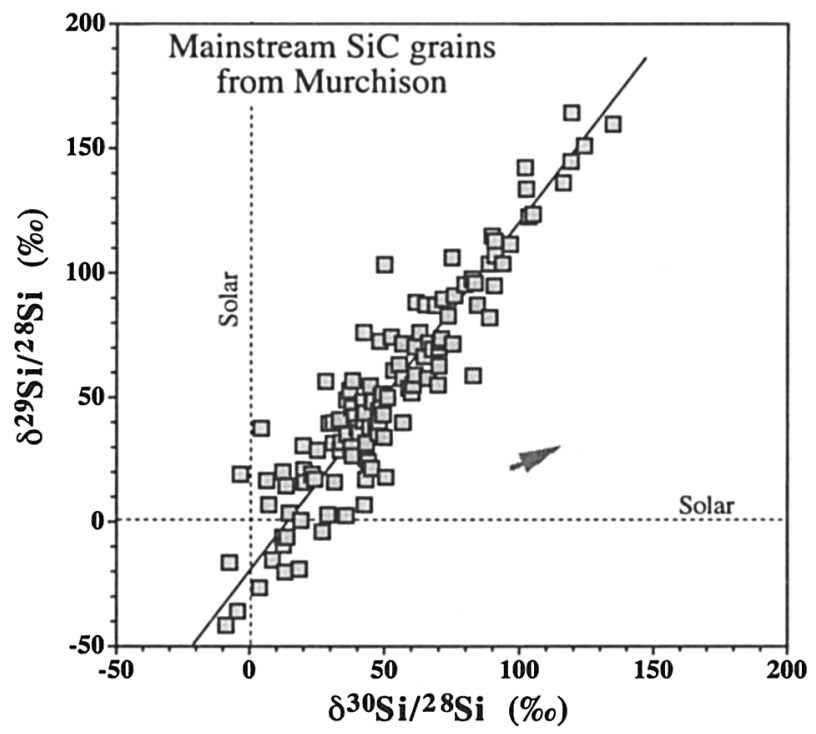

Figure 4. Silicon isotopic ratios measured in individual mainstream $\mathrm{SiC}$ grain (Hoppe et al. 1994). Ratios are plotted as $\delta$-values, deviations in permil from the solar isotopic ratios. The solid line is the slope 1.34 correlation line for mainstream $\mathrm{SiC}$ grains. The short arrow is the theoretically predicted shift in isotopic composition of the envelope of carbon stars that results from dredge-up of material that experienced neutron capture in the He shell.

1994; Brown \& Clayton 1992). Fig. 4 shows Si isotopic compositions measured in single $\mathrm{SiC}$ grains from the Murchison meteorite (Hoppe et al. 1994). The range of isotopic ratios is much larger than the shift expected from neutron capture, which is represented by the arrow, and thus cannot be explained by nucleosynthesis in a single AGB star. The most likely explanation is that many different AGB stars with a range in initial $\mathrm{Si}$ isotopic ratios contributed $\mathrm{SiC}$ to the Solar System (Clayton et al. 1991; Alexander 1993). This range in turn is attributed to galactic evolution and spatial heterogeneity of the $\mathrm{Si}$ isotopes (Timmes \& Clayton 1996; Clayton 1997; Clayton \& Timmes 1997; Alexander \& Nittler 1998; Lugaro et al. 1999). Similarly to the Si isotopes, the Ti isotopes in $\mathrm{SiC}$ seem to be affected by both neutron capture in AGB stars and galactic chemical evolution, however only a limited amount of data exists (Hoppe et al. 1994; Alexander \& Nittler 1998).

\section{Heavy elements in presolar $\mathrm{SiC}$}

All the heavy elements analyzed so far show the isotopic signature of the sprocess (Figs. 5 and 6). Elements measured in bulk samples include $\mathrm{Kr}$ and $\mathrm{Xe}$ (Lewis et al. 1994), as well as Sr (Podosek et al. 1998), Ba (Ott \& Begemann 1990; Zinner et al. 1991, Prombo et al. 1993), Nd and Sm (Zinner et al. 1991; Richter et al. 1993), and Dy (Richter et al. 1994). Recently, the development of micro-RIMS (resonance ionization mass spectrometry) has made it possible to 


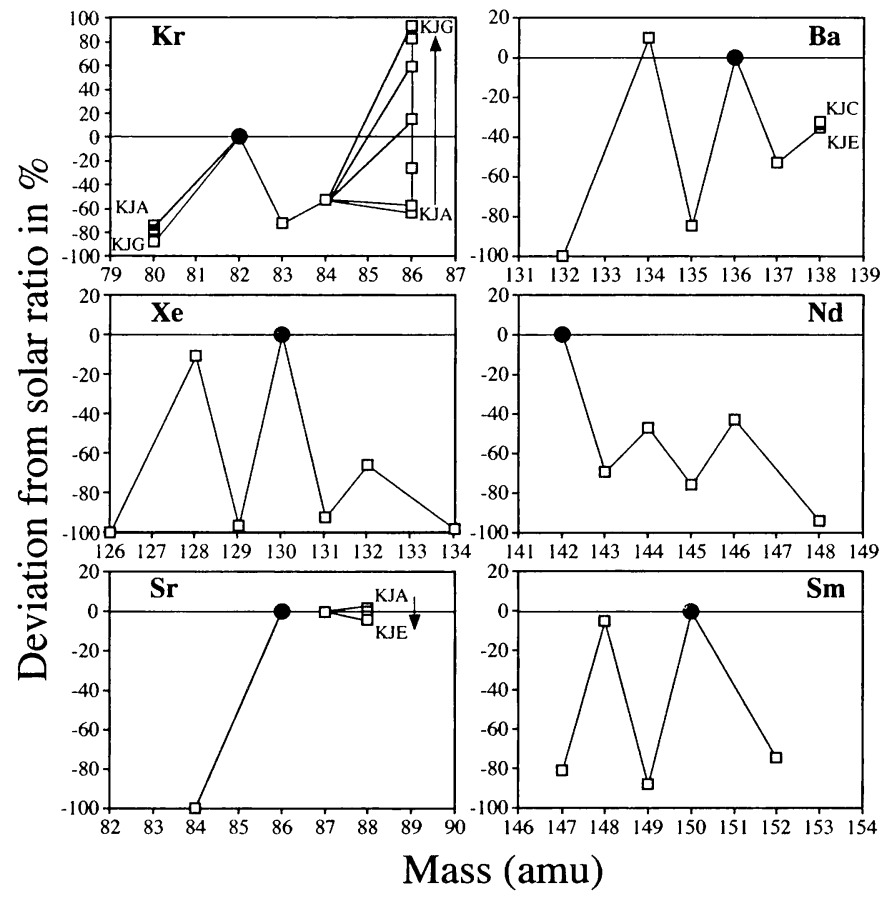

Figure 5. Isotopic patterns of heavy elements measured in bulk samples of $\mathrm{SiC}$ from the Murchison carbonaceous chondrite. Plotted are the s-process components extracted from the measurements (see Hoppe \& Ott 1997). Isotopic ratios are relative to the reference pure s-process isotopes plotted as solid circles and are normalized to the solar ratios. Different data points for the heaviest isotopes of $\mathrm{Kr}, \mathrm{Sr}$, and $\mathrm{Ba}$ are for different grains size fractions of $\mathrm{SiC}$; the sizes of their grains increase from $\mathrm{KJA}$ to $\mathrm{KJG}$.

measure Sr (Nicolussi et al. 1998a), Zr (Nicolussi et al. 1997) and Mo (Nicolussi et al. $1998 \mathrm{~b}$ ) in single $\mathrm{SiC}$ and graphite grains.

The measured isotopic compositions of heavy elements can be interpreted as a mixture of an s-process component (the $\mathrm{G}$ component), reflecting the composition of the He-shell of AGB stars, and the $\mathrm{N}$ component, reflecting the initial composition of the envelope and possible terrestrial contamination (see, e.g., Hoppe \& Ott 1997). The $\mathrm{G}$ components of $\mathrm{Kr}, \mathrm{Xe}, \mathrm{Sr}, \mathrm{Ba}, \mathrm{Nd}$, and $\mathrm{Sm}$ are plotted in Fig. 5. This component can be determined if a pure p- or r-process isotope can be measured. In each case the isotopic ratios are normalized to a s-only isotope and the solar ratio. With the exception of Dy (there might be still experimental problems) the s-process compositions of the heavy elements are well reproduced by the "classical model" of the main component (Käppeler et al. 1989) and by the refinements of AGB stellar models (Gallino et al. 1997). Discrepancies between grain data and earlier theoretical predictions for $\mathrm{Ba}$ and $\mathrm{Nd}$ (Gallino et al. 1993) could largely be resolved by improved measurements of 


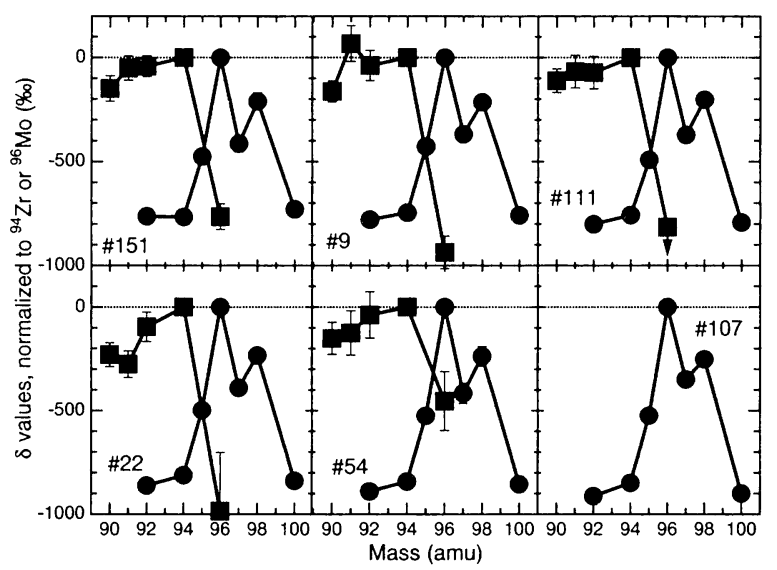

Figure 6. Zirconium (squares) and Mo (circles) isotopes measured by resonance ionization mass spectrometry in individual $\mathrm{SiC}$ grains from Murchison (Nicolussi et al. 1997, 1998b). Ratios are plotted as $\delta$-values, deviations in permil from the solar isotopic ratios. All grains shown have s-process isotopic patterns consistent with an origin in AGB stars. Figure courtesy of Andy Davis.

the relevant neutron capture cross sections (e.g., Voss et al. 1994, 1995, Wisshak et al. 1997).

The $\mathrm{SiC}$ data allow the determination of different s-process parameters. ${ }^{88} \mathrm{Sr} /{ }^{86} \mathrm{Sr}$ and ${ }^{138} \mathrm{Ba} /{ }^{136} \mathrm{Ba}$ ratios vary with grain size (Fig. 5), indicating differences in mean neutron exposure $\tau_{0}$. Inferred values for $\tau_{0}$ range from 0.14 to $0.17 \mathrm{mb}^{-1}$, only about half the value derived for the Solar System (Gallino et al. 1993). The large variation in the ${ }^{86} \mathrm{Kr} /{ }^{82} \mathrm{Kr}$ ratio among different grain size separates is attributed to differences in neutron density, which affects the branching point ${ }^{85} \mathrm{Kr}$ (Lewis et al. 1994).

The s-process signatures of $\mathrm{Zr}$ and Mo measured in single SiC grains (Fig. 6) are dominated by large ${ }^{96} \mathrm{Zr}$ depletions and by large depletions of the p-only isotopes ${ }^{92} \mathrm{Mo}$ and ${ }^{94} \mathrm{Mo}$ and of the r-only isotope ${ }^{100} \mathrm{Mo}$ (Nicolussi et al. 1998b). Intermediate depletions are found for the $\mathrm{s}+\mathrm{r}$ isotopes ${ }^{95} \mathrm{Mo},{ }^{97} \mathrm{Mo}$, and ${ }^{98} \mathrm{Mo}$. Since branching at ${ }^{95} \mathrm{Zr}\left(\mathrm{t}_{1 / 2} \approx 64\right.$ days) affects the production and destruction of ${ }^{96} \mathrm{Zr}$, its abundance sensitively depends on neutron density. Whereas at the low neutron densities expected from the ${ }^{13} \mathrm{C}(\alpha, \mathrm{n})$ source (Gallino et al. 1998$){ }^{96} \mathrm{Zr}$ is efficiently destroyed, activation of the ${ }^{22} \mathrm{Ne}(\alpha, n)$ source during late thermal pulses is predicted to restore some of this isotope. The virtual absence of ${ }^{96} \mathrm{Zr}$ in several grains means that the ${ }^{22} \mathrm{Ne}(\alpha, n)$ source was not active in their parent stars.

Acknowledgments. We thank Roberto Gallino for his continuing advice and Andy Davis for providing figure 6. This work was supported by NASA and NSF. 


\section{References}

Alexander C.M.O'D., 1993, Geochim. Cosmochim. Acta 57, 2869

Alexander C.M.O'D., Nittler L.R., 1998, ApJ submitted

Amari S., Nittler L.R., Zinner E., Lewis R.S., 1997, Met. \& Planet. Sci. 32, A6

Amari S., Lewis R.S., Anders E., 1995, Geochim. Cosmochim. Acta 53, 1411

Anders E., Zinner E., 1993, Meteoritics 28, 490

Bazan G., 1991, Ph.D. thesis, University of Illinois, Urbana-Champaign

Bernatowicz T., Fraundorf G., Ming T., Anders E., Wopenka B., Zinner E., Fraundorf P., 1987, Nature 330, 728

Bernatowicz T., Walker R.M., 1997, Physics Today 50 (12), 26

Bernatowicz T.J., Zinner E., 1997, Astrophysical Implications of the Laboratory Study of Presolar Materials, AIP, New York, p. 750

Boothroyd A.I., Sackmann I.-J., 1988, ApJ 328, 653

Boothroyd A.I., Sackmann I.-J., 1998, ApJ in press

Boothroyd A.I., Sackmann I.-J., Wasserburg G.J., 1994, ApJ 430, L77

Boothroyd A.I., Sackmann I.-J., Wasserburg G.J., 1995, ApJ 442, L21

Brown L.E., Clayton D.D., 1992, ApJ 392, L79

Charbonnel C., 1995, ApJ 453, L41

Choi B.-G., Huss G.R., Wasserburg G.J., 1998a, Met. \& Planet. Sci. 33, A32

Choi B.-G., Huss G.R., Wasserburg G.J., 1998b, Lunar Planet. Sci. XXIX, Abstract \#1898

Clayton D.D., 1997, ApJ 484, L67

Clayton D.D., Obradovic M., Guha S., Brown L.E., 1991, Lunar Planet. Sci. XXII, 221

Clayton D.D., Timmes F.X., 1997, ApJ 483, 220

Dearborn D.S.P., 1992, Phys. Rep. 210, 367

Denissenkov P.A., Weiss A., 1996, A\&A 308, 773

El Eid M., 1994, A\&A 285, 915

Forestini M., Paulus G., Arnould M., 1991, A\&A 252, 597

Frost C.A., Lattanzio J.C., 1996, in Stellar Evolution: What Should Be Done?, A. Noel, D. Fraipont-Caro, M. Gabriel, N. Grevesse \& P. Demarque (eds.), Université de Liège, Liège, p. 307

Gallino R., Arlandini C., Busso M., Lugaro M., Travaglio C., Straniero O., Chieffi A., Limongi M., 1998, ApJ 497, 388

Gallino R., Busso M., Lugaro M., 1997, in Astrophysical Implications of the Laboratory Study of Presolar Materials, T.J. Bernatowicz \& E. Zinner (eds.), AIP, New York, p. 115

Gallino R., Busso M., Picchio G., Raiteri C.M., 1990, Nature 348, 298

Gallino R., Raiteri C.M., Busso M., 1993, ApJ 410, 400

Gallino R., Raiteri C.M., Busso M., Matteucci F., 1994, ApJ 430, 858

Harris M.J., Lambert D.L., 1984, ApJ 285, 674

Harris M.J., Lambert D.L., Hinkle K.H., Gustafsson B., Eriksson K., 1987, ApJ 316, 294

Hoppe P., Amari S., Zinner E., Ireland T., Lewis R.S., 1994, ApJ 430, 870

Hoppe P., Kocher T.A., Strebel R., Eberhardt P., Amari S., Lewis R.S., 1996, Lunar Planet. Sci. XXVII, 561 
Hoppe P., Ott U., 1997, in Astrophysical Implications of the Laboratory Study of Presolar Materials, T.J. Bernatowicz \& E. Zinner (eds.), AIP, New York, p. 27

Huss G.R., Hutcheon I.D., Wasserburg G.J., 1997, Geochim. Cosmochim. Acta 61, 5117

Hutcheon I.D., Huss G.R., Fahey A.J., Wasserburg G.J., 1994, ApJ 425, L97

Iben I., Jr., Renzini A., 1983, ARA\&A 21, 271

Käppeler F., Beer H., Wisshak K., 1989, Rep. Prog. Phys. 52, 945

Lattanzio J.C., Boothroyd A.I., 1997, in Astrophysical Implications of the Laboratory Study of Presolar Materials, T.J. Bernatowicz \& E. Zinner (eds.), AIP, New York, p. 85

Lewis R.S., Amari S., Anders E., 1990, Nature 348, 293

Lewis R.S., Amari S., Anders E., 1994, Geochim. Cosmochim. Acta 58, 471

Lewis R.S., Tang M., Wacker J.F., Anders E., Steel E., 1987, Nature 326, 160

Little-Marenin I.R., 1986, ApJ 307, L15

Lugaro M., Gallino R., Busso M., Amari S., Zinner E., 1999, in Nuclei in the Cosmos V, N. Prantzos (ed.), in press

Nicolussi G.K., Davis A.M., Pellin M.J., Lewis R.S., Clayton R.N., Amari S., 1997, Science 277, 1281

Nicolussi G.K., Pellin M.J., Lewis R.S., Davis A.M., Clayton R.N., Amari S., 1998a, Phys.Rev.Lett in press

Nicolussi G.K., Pellin M.J., Lewis R.S., Davis A.M., Clayton R.N., Amari S., 1998b, ApJ 504, 492

Nittler L.R., 1997, in Astrophysical Implications of the Laboratory Study of Presolar Materials, T.J. Bernatowicz \& E. Zinner (eds.), AIP, New York, p. 59

Nittler L.R., Alexander C.M.O'D., Gao X., Walker R.M., Zinner E., 1997, ApJ 483, 475

Nittler L.R., Alexander C.M.O'D., Wang J., Gao X., 1998, Nature 393, 222

Nittler L.R., Hoppe P., Alexander C.M.O'D., Amari S., Eberhardt P., Gao X., Lewis R.S., Strebel R., Walker R.M., Zinner E., 1995, ApJ 453, L25

Ott U., 1993, Nature 364,25

Ott U., Begemann F., 1990, ApJ 353, L57

Podosek F.A., Prombo C.A., Amari S., Lewis R.S., 1998, ApJ in press

Prombo C.A., Podosek F.A., Amari S., Lewis R.S., 1993, ApJ 410, 393

Richter S., Ott U., Begemann F., 1993, in Nuclei in the Cosmos, F. Käppeler \& K. Wisshak (eds.), Institute of Physics, Bristol and Philadelphia, p. 127

Richter S., Ott U., Begemann F., 1994, in Proc. Europ. Workshop on Heavy Element Nucleosynthesis, E. Somorjai \& Z. Fülöp (eds.), Hung. Acad. Sci., Debrecen, p. 44

Smith V.V., Lambert D.L., 1990, ApJS 72, 387

Tang M., Lewis R.S., Anders E., Grady M.M., Wright I.P., Pillinger C.T., 1988, Geochim. Cosmochim. Acta 52, 1221

Timmes F.X., Clayton D.D., 1996, ApJ 472, 723

Timmes F.X., Woosley S.E., Weaver T.A., 1995, ApJS 98, 617

Voss F., Wisshak K., Guber K., Käppeler F., Reffo G., 1994, Phys.Rev.C 50, 2582

Voss F., Wisshak K., Käppeler F., 1995, Phys.Rev.C 52, 1102

Wasserburg G.J., Boothroyd A.I., Sackmann I.-J., 1995, ApJ 447, L37

Wisshak K., Voss F., Käppeler F., Kazakov L., 1997, Nucl. Phys. A621, 270c

Zinner E., 1998, Ann. Rev. Earth \& Planet. Sci. 26, 147

Zinner E., Amari S., Lewis R.S., 1991, ApJ 382, L47 